\title{
THz Reflection Spectra of Different Materials, Including Explosives, Measured at a Distance up to $5 \mathrm{~m}$
}

\author{
M. Walczakowski ${ }^{a}$, N. Palka ${ }^{a}$, A. Czerwiński ${ }^{b}$, M. Sypek $^{b}$ And M. Szustakowski ${ }^{a}$ \\ ${ }^{a}$ Institute of Optoelectronics, Military University of Technology, S. Kaliskiego 2, 00-908 Warszawa, Poland \\ ${ }^{b}$ Warsaw University of Technology, Koszykowa 75, 00-662 Warszawa, Poland
}

(Received May 12, 2014)

\begin{abstract}
This work presents a terahertz system designed for the reflection spectroscopy of different materials located at a distance up to $5 \mathrm{~m}$. The source of the radiation is a tunable solid-state optical parametric oscillator, which generates a narrow-band nanosecond pulses in the range of $0.7-2.5 \mathrm{THz}$. The signal is detected with relatively fast and having big sensitivity hot electron bolometer. The detailed description of each device and the functioning of the experimental setup are provided as well as the methodology of the measurement is explained. Investigations were performed in the $0.7-2.2 \mathrm{THz}$ range in free space with relative humidity of about $40 \%$. The experiment was divided into three series, each of which was carried out with different distance between the examined sample and the system $-1 \mathrm{~m}, 3 \mathrm{~m}$, and $5 \mathrm{~m}$. Obtained spectra of selected materials, including explosives, are similar to the results received from a purged time domain spectroscopy system. The observed small deviations are connected with fluctuations of the laser wavelength and the instability of the bolometer.
\end{abstract}

DOI: $10.12693 /$ APhysPolA.127.689

PACS: 06.60.Ei, 07.57.Hm, 07.57.Kp, 07.57.Pt, 33.20.Ea

\section{Introduction}

Many concerns about the worldwide public safety caused new law regulations enforcing security improvements. Besides the improved security requirements an important aspect of the safety and the health of people being scanned became crucial issue. This fact gave the contribution to the significant increase in the interest of remote sensing applications in recent years. Latest developments in the terahertz techniques make it possible to take the advantage of the unique properties of the radiation in far infrared spectrum. Such radiation can be applied to sensing systems design like passive sub- $\mathrm{THz}$ cameras for hidden object detection [1] or multispectral explosives sensors [2]. We present the experimental setup capable of remote identification of hazardous and dangerous materials in the $\mathrm{THz}$ band (up to $5 \mathrm{~m}$ distance detection range). The examined setup is the modified and improved version of the one used in our previous research [3]. The source of the radiation is a commercially available optical parametric oscillator (OPO). Its spectral working range is $0.7-2.5 \mathrm{THz}$ with the $53 \mathrm{~Hz}$ pulse repetition rate. Duration of the pulse have FWHM at about $20 \mathrm{~ns}$ with $10 \mathrm{~nJ}$ energy, and the pulse peak power around $10 \mathrm{~W}$. The generated $\mathrm{THz}$ beam is then optically formed and directed by a flat mirror to the target. The radiation reflected back from the examined sample propagates back in the free space atmosphere and then passes through a set of mirrors reaching the detector (hot electron bolometer, HEB).

\section{Experimental setup}

The experimental setup (Fig. 1) was designed in the manner to enable carrying out measurements at different distances between the sample and the system. Additionally, the sample can be placed with up to $1 \mathrm{~m}$ in-plane divergences without making changes to the optical setup, only minor mirror adjustments are necessary.

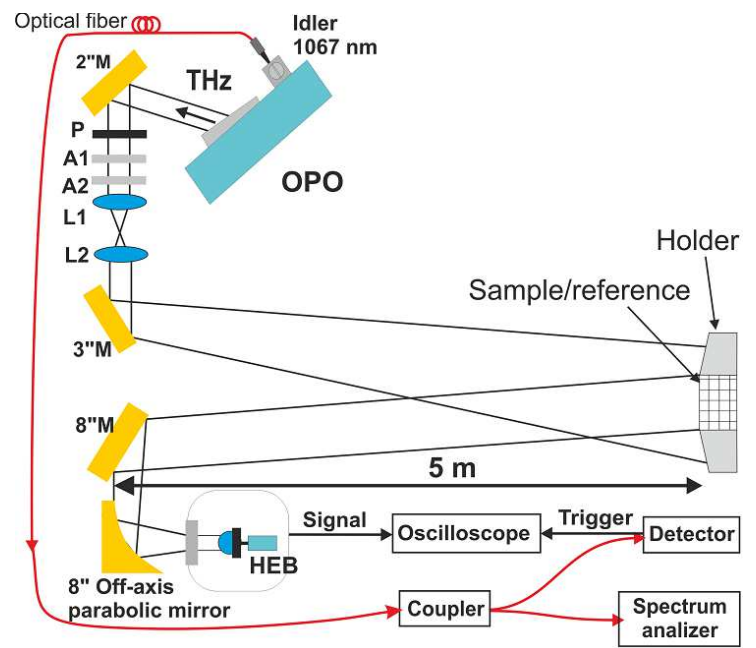

Fig. 1. The scheme of the experimental setup.

The sample handling is very important and has significant influence on the obtained spectra. Pure materials in the transmission spectroscopy have too high attenuation in the $\mathrm{THz}$ range. Exactly the opposite situation takes place in the reflection spectroscopy. In this case material without dopants gives higher absorption changes in output spectra. We used pellets with the diameter of $30 \mathrm{~mm}$ and their thickness was about $3 \mathrm{~mm}$. To prevent mixing the signal reflected from the sample with the one scattered on the holder we build a round shape holder with angle cut surface (Fig. 2). Mixing those two signals would result in the degradation of the $\mathrm{S} / \mathrm{N}$ ratio. To eliminate any influence of the backscattered radiation on the registered spectrum, the holder is made from metal with polished surface. The flat sample is placed in the middle 
of the holder so that it provides us with certainty that all unwanted radiation will definitely not reach the detector.

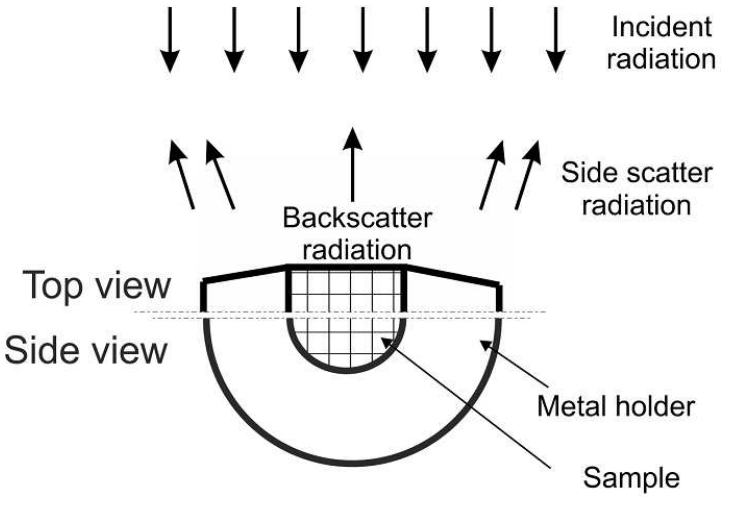

Fig. 2. The schematic diagram of the sample holder.

The source of the $\mathrm{THz}$ radiation is the solid-state laser delivered by MSquare [4]. The laser is working in the range of $0.7-2.5 \mathrm{THz}$ with $53 \mathrm{~Hz}$ repetition rate and is based on the OPO [5] phenomenon. Duration of the pulse is about $20 \mathrm{~ns}$ with $50 \mathrm{GHz}$ band and $10 \mathrm{~nJ}$ energy. The pulsed operation is achieved by the modulation of the cavity $Q$ factor. The nonlinear lithium niobate crystal doped with the magnesium oxide $\left(\mathrm{MgO}: \mathrm{LiNbO}_{3}\right)$ is placed in an optical resonant cavity, which is also an active region. The pumping signal incidents on the crystal and then it is converted into two signals with lower frequencies according to the relationship [6]:

$$
\omega_{\mathrm{p}}=\omega_{\mathrm{i}}+\omega_{\mathrm{THz}}
$$

where $\omega_{\mathrm{p}}$ is the pumping frequency, $\omega_{\mathrm{i}}$ is the idler signal and $\omega_{\mathrm{THz}}$ is the terahertz signal frequency.

The OPO generates two usable signals: the optical idler signal and the terahertz signal. The idler pulse, with the wavelength of about $1067 \mathrm{~nm}$, is coupled into the optical fiber. After splitting it by the coupler, one part is used as a trigger and the second one is measured by the spectrum analyzer. We use formula (1) to determine the frequency of the terahertz signal by measuring idler frequency. The graphical interpretation of the noncollinear phase matching is shown in Fig. 3.

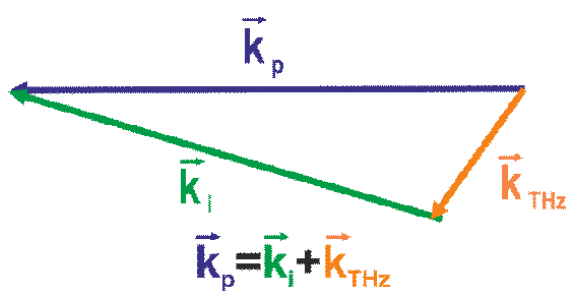

Fig. 3. The non-collinear phase matching.

The possibility of laser tuning is obtained by changing the conditions of the interaction of the pumping signal with the crystal which results in simultaneous tuning of the differential and the terahertz signals. The tuning range is limited only by absorption and reflection of the crystal inside the resonator. The generated $\mathrm{THz}$ beam is slightly divergent with a diameter of about $20 \mathrm{~mm}$. It is collimated by two 2" concavo-plane polyethylene lenses (L1, L2) having $15 \mathrm{~mm}$ focal lengths (but small divergence of the beam is desired). The beam passes through the polarizer $(\mathrm{P})$, which provides linear polarization of the radiation relevant in the spectroscopy of some materials. Due to the low dynamic range of the detector, the $\mathrm{THz}$ beam is attenuated by a set of attenuators (A1, A2), in some cases even up to factor of $10^{5}$. Afterwards, the beam is directed by a 3 " flat mirror (M) to the target the flat $30 \mathrm{~mm}$ steel disc which serves as a reference in the experiment. Only the radiation from the disc is reflected back, it propagates $5 \mathrm{~m}$ through the atmosphere and is directed to the detector through the flat 8 " mirror and the 8 " off-axis parabolic mirror. During measurements on distances shorter than $3 \mathrm{~m}$ substituting 4" mirrors for 8 " ones did not make any noticeable differences in obtained spectra. The incidence angle of the beam on the target surface is about $3^{\circ}$.

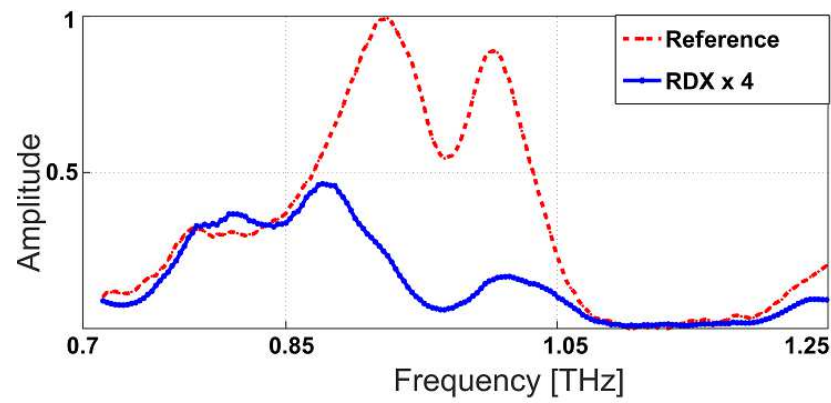

Fig. 4. The signal reflected from the sample and the reference.

The beam is detected by relatively fast and having large sensitivity hot electron bolometer [7] operating at $8 \mathrm{~K}$ thanks to the pulse tube cryocooler. The sensing component is a $4 \mathrm{~nm}$ thick bridge made of the niobium nitride $(\mathrm{NbN})$ integrated with a planar logarithmic spiral antenna on a substrate of highly resistive silicon [8]. The aperture of the detector input is limited to less than $3^{\circ}$. Before the terahertz signal reaches the bolometer, it passes through the window made of the high density polyethylene (HDPE) and infrared band pass filter limiting the operation range to $0.3-3.0 \mathrm{THz}$. The filtered signal is collected by a silicon lens and focused on the bridge. The spectral response measurement of the material is carried out by tuning the laser and collecting data point for each frequency. Due to the instability of the pulses duration and their position at different frequencies, information about the spectral properties of the test material is determined on the basis of the pulse energy. The pulse energy is calculated by integrating the signal envelope in the appropriate limits. The measurement uncertainty of each point is reduced by averaging at least 30 pulses. The reflectance of the sample is obtained by comparing the reference and the sample measurements (Fig. 4) according to the formula 


$$
\mathrm{R}(\mathrm{f})=\mathrm{I}_{1}(\mathrm{f}) / \mathrm{I}_{0}(\mathrm{f}),
$$

where $I_{1}(f)$ is the sample measurement and $I_{0}(f)$ is the reference measurement.

The signal losses (about 30-40 dB) are mainly associated with the attenuation of the atmosphere and the existing mismatch between the beam and the disc diameters. Despite such heavy losses, the $\mathrm{THz}$ signal detection in this setup was possible with $\mathrm{S} / \mathrm{N}$ ratio even up to $21 \mathrm{~dB}$ which reveals the great potential of stand-off detection of different materials.

\section{Results and discussion}

Figures 5 to 8 present results of measurements of hexogen (RDX), PABA and lactose samples in distances of $1 \mathrm{~m}, 3 \mathrm{~m}$, and $5 \mathrm{~m}$ from the setup. These results were compared to spectra measured with the time domain spectroscopy system [9]. The TDS results were obtained in dry atmosphere with humidity less than $1 \%$. The OPO-HEB setup worked in the open space atmosphere at room temperature with humidity of about $40 \%$.

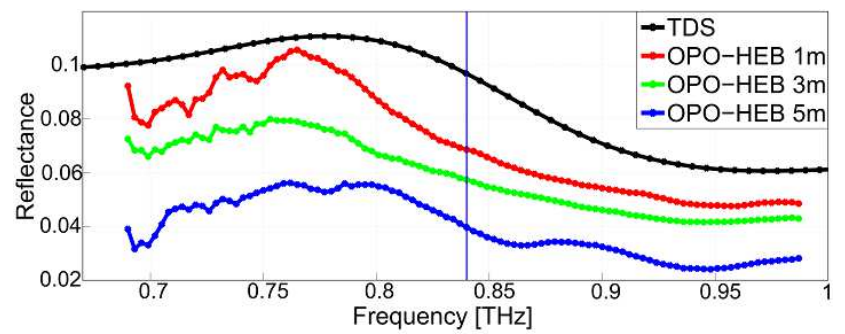

Fig. 5. $\mathrm{THz}$ reflectance spectra of the RDX in $0.67-1 \mathrm{THz}$ range.

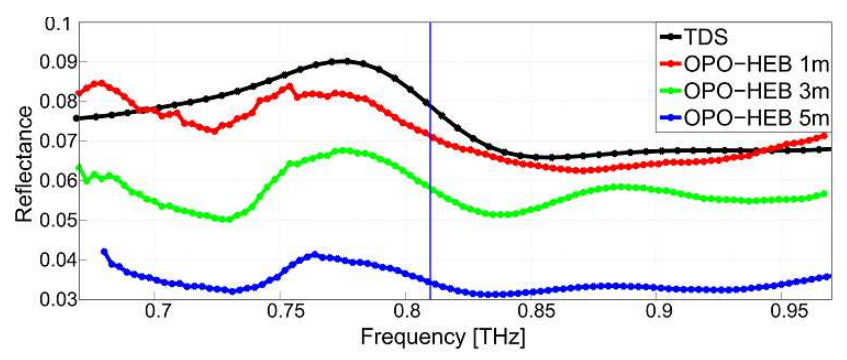

Fig. 6. $\mathrm{THz}$ reflectance spectra of the PABA in $0.67-1 \mathrm{THz}$ range.

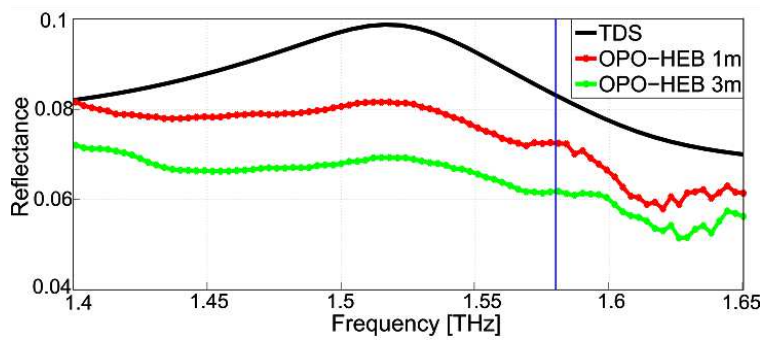

Fig. 7. $\mathrm{THz}$ reflectance spectra of the PABA in 1.4-1.65 THz range.

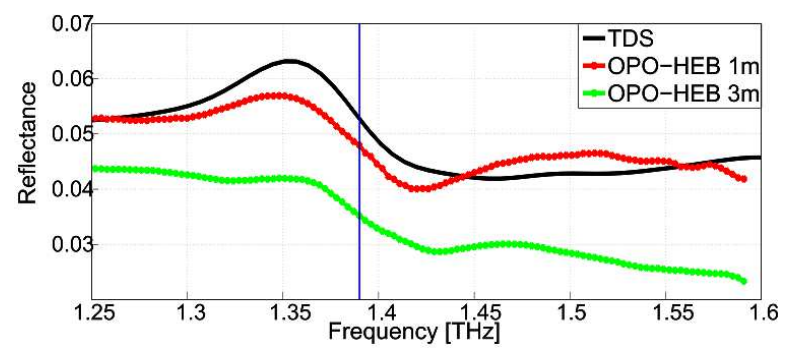

Fig. 8. $\mathrm{THz}$ reflectance spectra of the Lactose in 1.25-1.6 $\mathrm{THz}$ range.

This fact implies the presence of water vapor absorption peaks in the measured characteristics. Since the material and reference spectra were measured in the similar atmospheric conditions, the water vapor influence on the absorption spectra of materials should be eliminated. However, we can notice small absorption features at frequencies corresponding to the water vapor absorption. This fact can be explained by different configuration of the attenuators used for the reference and the sample measurements. Taking into account limiting the dynamic range to $21 \mathrm{~dB}$ every water vapor absorption line exceeding $21 \mathrm{~dB}$ is noticeable. Also it must be noticed that the emission characteristic of the source is nonlinear, therefore, in some frequency regions the dynamics of the setup was limited and in these regions we can observe even weaker water vapor absorption lines. Such situation takes place around the lower frequency limit of the OPO $(0.7 \mathrm{THz})$ and can be observed in Fig. 5 and Fig. 6.

The considered OPO-HEB setup shows good agreement of the spectral characteristics in comparison to the time domain spectroscopy (TDS) method.

\section{Conclusions}

We report on the terahertz reflectance spectra of three materials: RDX, PABA, and lactose, measured in the atmosphere with about $40 \% \mathrm{RH}$. The penetration of soft materials and clothing by the terahertz waves makes it possible to identify materials hidden underneath or masked in a different manner. The obtained results and the performance of the presented setup show that further research is needed to determine what are the possibilities and the limitations of presented measuring techniques. Despite some undisputed advantages, the $\mathrm{THz}$ technology is rather bulky and difficult for practical uses. Special systems are used in predesigned environment, like airports or government facilities, but applications in broad range of public safety are limited. All that should be an imperative for constant equipment development. The presented setup has some limitations but not all of them are caused by using the terahertz reflection spectroscopy. The water vapor absorption in the free space is undoubtedly the biggest problem during the measurement. Moreover, some negative influence of water vapor on the registered signal can be reduced by data post processing. Other limitations can be overcome by further development of the equipment. Two most obvious 
disadvantages of the designed setup are too low repetition rate of the radiation source and limited dynamic range of the detector. The dynamic range of the detector could be improved by replacing it by a pyroelectric one, but this leads to even 1000 times drop in sensitivity.

\section{References}

[1] M. Kowalski, N. Palka, M. Piszczek, M. Szustakowski, Acta Phys. Pol. A. 124, 490 (2013).

[2] J. Wojtas, T. Stacewicz, Z. Bielecki, B. Rutecka, R. Medrzycki, J. Mikolajczyk, Opto-electron. Rev. 21, 210 (2013).

[3] N. Pałka, M. Walczakowski, P. Zagrajek, A. Czerwiński, M. Sypek, M. Szustakowski, Acta Phys. Pol. A 124, 538 (2013).
[4] Firefly-THz User Manual, MSquared Lasers Ltd, Glasgow 2011.

[5] J.-I. Shikata, M. Sato, T. Taniuchi, H. Ito, K. Kawase, Opt. Lett. 24, 202 (1999).

[6] D. Molter, M. Theuer, R. Beigang, Opt. Expr. 17, 6623 (2009).

[7] HEB User Manual, Scontel Superconducting Nanotechnology, Moscow 2011.

[8] A.D. Semenov, H.-W. Hubers, J. Schubert, G.N. Gol'tsman, A.I. Elantiev, B. Voronov, E.M. Gershenzon, J. Appl. Phys. 88, 6758 (2000).

[9] L. Dexheimer, Terahertz Spectroscopy: Principles and Applications, CRC Press, Boca Raton 2008. 